\title{
COMPOSICIÓN FLORÍSTICA Y ESTADO DE CONSERVACIÓN DE PLANTAS VASCULARES DEL DISTRITO DE CAJATAMBO / LIMA / PERÚ
}

\section{FLORISTIC COMPOSITION AND STATE OF CONSERVATION OF VASCULAR PLANTS OF THE DISTRICT OF CAJATAMBO / LIMA / PERU}

\author{
Aldo Ceroni Stuva ${ }^{1}$ y Graciela Vilcapoma Segovia ${ }^{2}$
}

\section{Resumen}

Se estudiaron la composición florística y el estado de conservación de las plantas amenazadas en Cajatambo / Lima / Perú. Las plantas fueron colectadas en alturas que oscilaron entre los 2802 y 4534 msnm en nueve formaciones vegetales. Se reportan 64 familias, 211 géneros y 355 especies, siendo Asteraceae, Poaceae, Fabaceae, Caryophyllaceae, Lamiaceae y Solanaceae las familias mejor representadas, y los géneros con mayor número de especies Senecio, Baccharis, Calceolaria, Oxalis y Solanum. Se encontraron 31 especies en alguna categoría de amenaza. Algunas especies están presentes sólo en un lugar como en Ocopata, Iscu-Viconga, Huaylashtoclanca y en el cerro San Cristóbal. Las formaciones vegetales con mayor presencia de especies amenazadas fueron: matorral, campo agrícola, pajonal y bosque de Polylepis.

Palabras clave: composición florística, formación vegetal, grado de amenaza.

\begin{abstract}
Floristic composition and state of conservation of the threatened plants in Cajatambo / Lima / Peru were studied. The plants were collected at altitudes that ranged between 2802 and 4534 masl, in nine plant formations. A total number of 64 families, 211 genera and 355 species were reported, being Asteraceae, Poaceae, Fabaceae, Caryophyllaceae, Lamiaceae and Solanaceae the families best represented, and the genera with the highest number of species were Senecio, Baccharis, Calceolaria, Oxalis and Solanum. It was found out that 31 species belong to some category of threat. Some species are present only in a place such as Ocopata, Iscu-Viconga, Hualylashtoclanca and San Cristobal hill. The plant formations with higher presence of threatened species were: scrubland, agricultural field, grassland and Polylepis forest.
\end{abstract}

Key words: floristic composition, plant formation, degree of threat.

\section{Introducción}

La flora es el conjunto de especies vegetales de un país o región, mientras que la vegetación es el tapiz vegetal conformado por las formas biológicas de las plantas (Font Quer, 1965). La flora y la vegetación brindan información relevante sobre la composición, estructura y funcionamiento del ecosistema (ONERN, 1975; Matteucci \& Colma, 1982; Brack \& Mendiola, 2004; Gutiérrez \& Squeo, 2004; De la Cruz et al., 2005; León et al., 2006). Los estudios florísticos que se llevan a cabo en los ecosistemas andinos desempeñan un papel importante en los trabajos de conservación y sistemática, ya que permiten conocer tanto la distribución geográfica de las plantas, como asî también su presencia en ciertas áreas (Aquino et al., 2017). Para la Región Lima, y lugares cercanos a Cajatambo, se cuenta con algunos estudios florísticos como los realizados en el Distrito de Oyón (Paulino et al., 2015; Kahn et al., 2016), Santa Rosa de Quives (Gonzáles et al., 2015), la Reserva Paisajística Nor
Yauyos-Cochas (Trinidad \& Cano, 2016), el anexo de Marachanca en Matucana (Aquino et al., 2017), y de Huarochirí (Aquino et al., 2018). Asimismo, los realizados en la Región Ancash como en el Parque Nacional Huascarán (Smith, 1988), Huari (Cano et al., 2005), Callejón de Conchucos (Cano et al., 2006), y en la Cordillera Blanca (Cano et al., 2010). En la sierra de Lima, por encima de los 1000 msnm, se encuentran, en general, diversas especies xerofíticas, plantas suculentas con metabolismo CAM, arbustos caducifolios y hierbas anuales (Weberbauer, 1945; ONERN, 1975). Estudios realizados sobre cactáceas y suculentas en los valles de Cañete, Chancay, Chillón, Huaura y Pativilca, también resaltan la importancia de estos tipos de plantas en la sierra de Lima, tanto en la configuración de la estructura vegetal como en su rol de eje de interacciones con otros elementos del sistema (Ostolaza et al., 2003, 2005, 2006, 2007, 2009; Ceroni et al., 2007). Estos estudios permiten conocer no solo la diversidad florística, sino también, la distribución, 


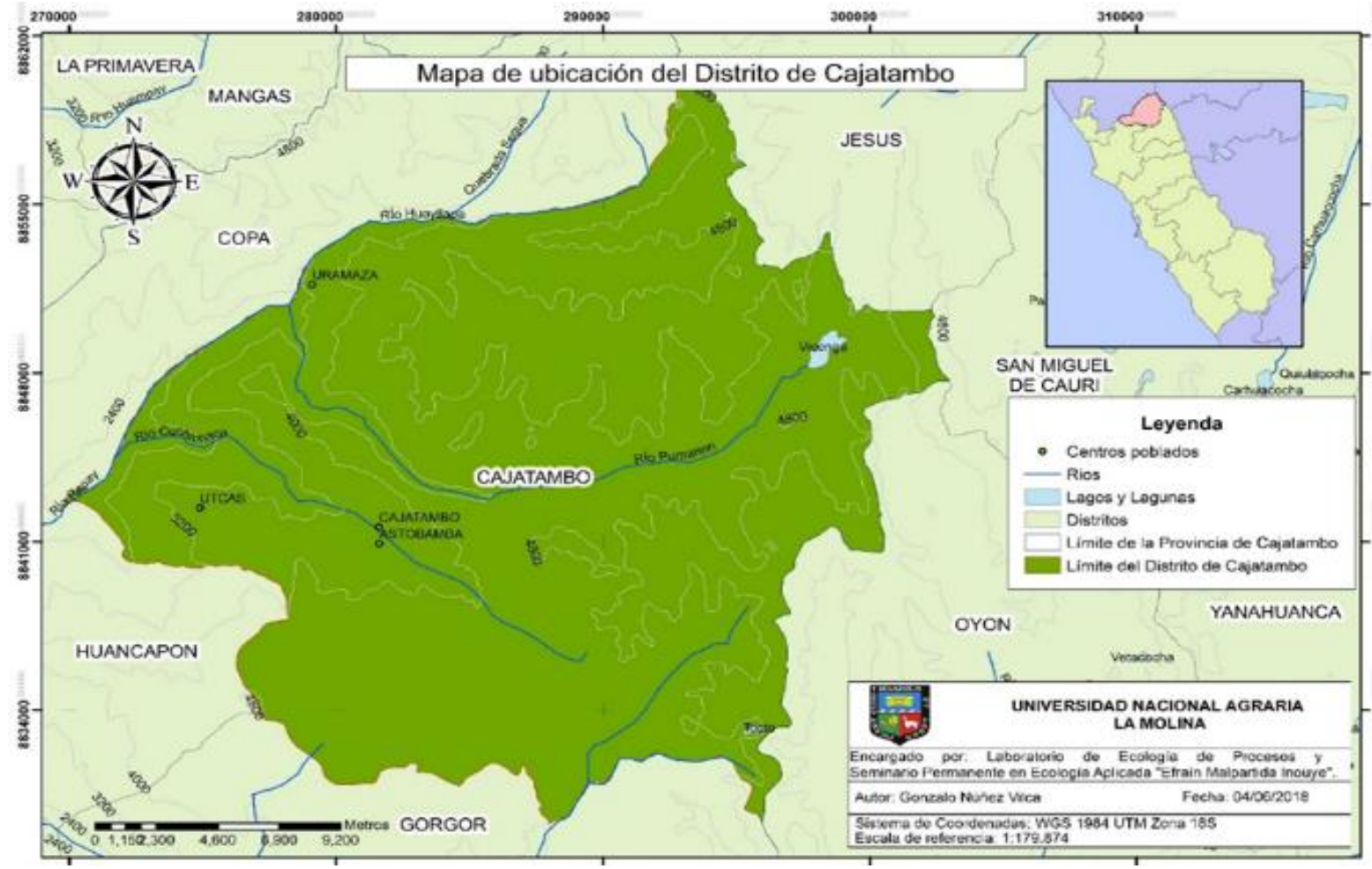

Figura 1. Ubicación del Distrito de Cajatambo en la Región Lima, Perú.

los endemismos y las especies amenazadas. Sin embargo, a pesar de los estudios florísticos y de la caracterización de la vegetación realizados y documentados en guías de campo, en algunas regiones del país con ecosistemas de montañas (mayor a los 3000 - $4000 \mathrm{msnm}$ ) en los Andes Peruanos, aún no existe información (Kahn et al., 2016) o el grado de conocimiento es todavía limitado (Ramos et al., 2015). Para Cajatambo se cuenta solo con estudios etnobotánicos, como los de Quinteros (2009) sobre los conocimientos tradicionales de la flora, en donde se da a conocer un total de 200 especies para el lugar, y el de Benavides (2019) sobre usos tradicionales de la diversidad vegetal. También se presenta una situación similar a la expresada por Cano et al. (2011) para los altos Andes, en el sentido de ser Cajatambo una zona con vacíos de información botánica y un ecosistema donde se desarrollan importantes actividades humanas que pueden impactar en la flora y la vegetación. La flora de Cajatambo también incluye especies consideradas con diferentes grados de amenaza. Quinteros (2009), al evaluar y muestrear siete localidades de Cajatambo, encontró varias especies vegetales citadas en el Anexo 1 del Decreto Supremo $\mathrm{N}^{\circ}$ 043-2006-AG (MINAGRI, 2006). De las 12 especies que se encuentran categorizadas como flora amenazada, 11 de ellas son conocidas por los cajatambinos y son utilizadas, de alguna manera, como medicina, leña, alimento, etc. Las especies de cactáceas y suculentas también están representadas en la vegetación de Cajatambo, algunas de las cuales se encuentran categorizadas En Peligro o Vulnerables
(Ostolaza et al., 2006). En este contexto, el objetivo del presente trabajo fue contribuir al conocimiento de la flora del Distrito de Cajatambo a través de dos objetivos específicos: un registro de la composición florística y una actualización del estado de conservación de las plantas amenazadas.

\section{Materiales y Métodos}

Ubicación del área de estudio

El área de estudio está ubicada en el Distrito de Cajatambo, en la provincia del mismo nombre, al NE del Departamento de Lima / Perú, en la parte occidental de la cordillera andina, a $10^{\circ} 28^{\prime} 16.65^{\prime \prime} \mathrm{LS}$ y $76^{\circ} 59^{\prime} 35.91^{\prime \prime}$ LO, limitada por los departamentos de Ancash, Huánuco y Pasco (Figura 1), a una elevación de entre los 2600 y $4800 \mathrm{msnm}$. La capital, Cajatambo, se encuentra a $3376 \mathrm{msnm}$ (Quinteros, 2009). Según datos de precipitación (mm) de la Estación Meteorológica Convencional de Cajatambo, para el periodo 2015 - 2016, los meses de lluvia se concentraron entre enero y abril, mientras que los meses secos entre junio y setiembre (Castro, 2019).

$\underline{\text { Registro de la composición florística }}$

Se colectó material botánico en abril y setiembre de los años 2015 y 2016, es decir, al final de las temporadas lluviosa y seca, respectivamente. A fin de tener una mayor representación de las especies, la recolección se realizó dentro de 44 transectos de $30 \mathrm{~m}$. de longitud por $20 \mathrm{~cm}$. de ancho, ubicados en el mosaico de parches o formaciones vegetales, determinado por fotointerpretación (Arnao, 2017) y a lo largo de los desplazamientos en el área de estudio 
(Figura 2). Las formaciones vegetales evaluadas fueron nueve: campo agrícola, bosque de eucalipto, bosque de Polylepis, matorral, pajonal, césped, bofedal, gravilla y juncal. El número de transectos fue proporcional al tamaño de cada tipo de parche y la accesibilidad al lugar (Tabla 1). La colección botánica fue hecha de acuerdo a la técnica de colección convencional (Smith, 1971; Mori et al., 1985; Lot \& Chiang, 1986; Young \& León, 1990), mientras que el prensado definitivo y secado se realizó en el Centro de Interpretación Casa Julio Gaudron del Jardín Botánico “Octavio Velarde Núñez" de la Universidad Nacional Agraria La Molina. El sistema de clasificación utilizado fue APG IV (APG, 2016) y las muestras herborizadas fueron depositadas en el Herbario del Jardín Botánico "Octavio Velarde Núñez” de la Universidad Nacional Agraria La Molina. Estado de las plantas amenazadas

La actualización del estado de las plantas amenazadas se hizo comparando las especies determinadas en el presente registro con los listados de especies amenazadas de la Unión Internacional para la
Conservación de la Naturaleza (UICN) en su "Lista Roja" versión 2018-2 (UICN, 2018), el Listado de Especies de Flora Silvestre CITES - Perú (CITES, 2017) y las evaluaciones de presencia, distribución y estado de conservación de cactáceas y suculentas en Lima según Ostolaza (Ostolaza et al., 2003, 2005, 2006, 2007, 2009; Ostolaza, 2014).

\section{Resultados y discusión}

$\underline{\text { Registro de la composición florística }}$

Se determinaron 355 especies, correspondientes a 211 géneros y 64 familias (Tabla 2). Las familias con mayor número de especies fueron: Asteraceae, Poaceae, Fabaceae, Caryophyllaceae, Lamiaceae y Solanaceae (Figura 3); mientras que los géneros con mayor número de especies fueron: Senecio, Baccharis, Calceolaria, Oxalis y Solanum, con 16, 9, 9, 7 y 7 especies, respectivamente (Tabla 2). Con respecto a trabajos realizados por Quinteros (2009), se encuentran coincidencias en cuanto a las familias botánicas dominantes, como Asteraceae, Lamiaceae, Fabaceae,

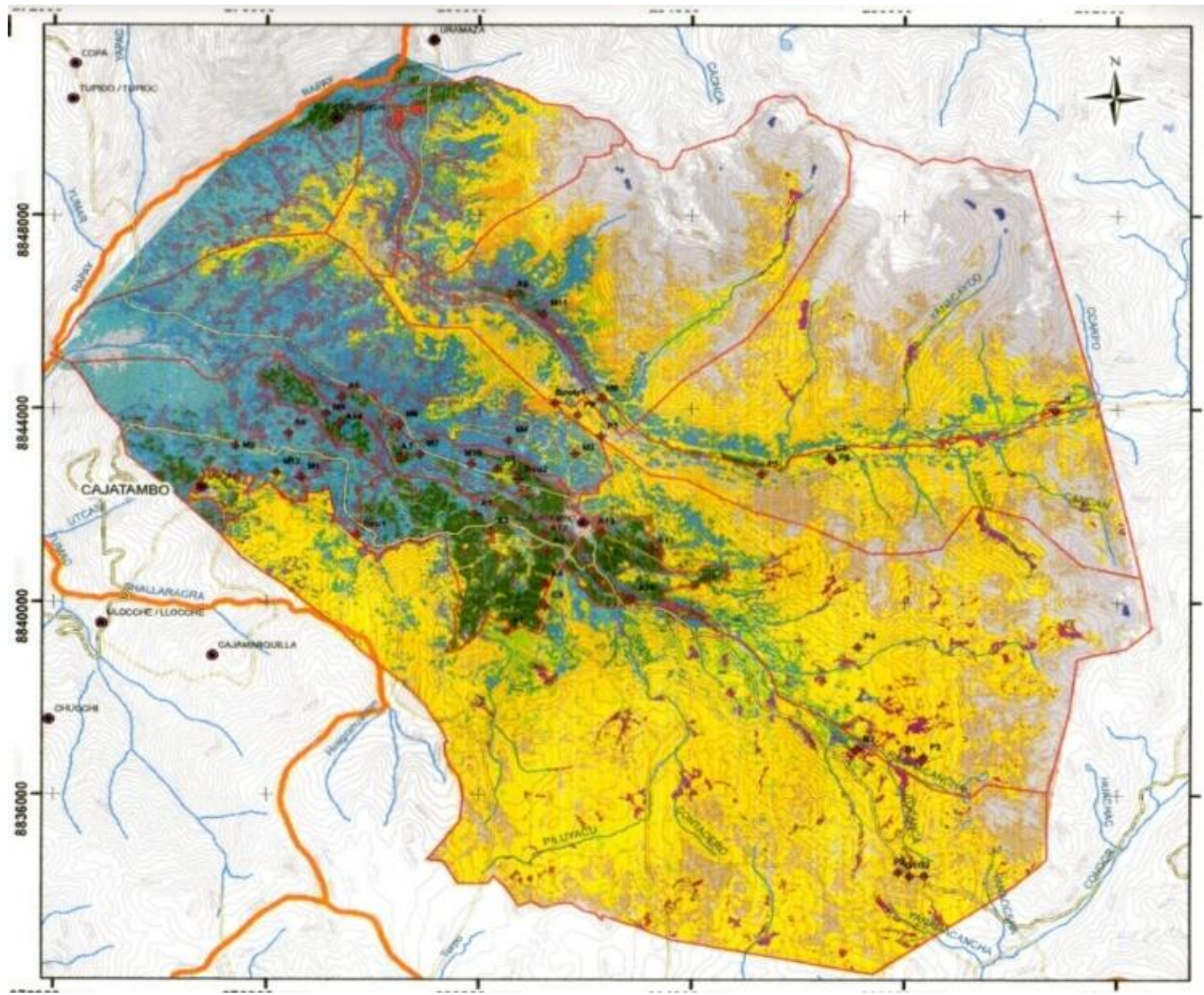

Figura 2. Mosaico de parches de la vegetación del Distrito de Cajatambo en la Región Lima, donde se destaca las tres formaciones vegetales más extensas: Pajonal en amarillo, Matorral en azul y Campo agrícola en verde. (Fuente: Arnao, 2017). 
Rosaceae, Poaceae, Brassicaceae y Solanaceae, a excepción de Scrophulariaceae, que ahora está desmembrada en otras familias. Otros trabajos realizados en zonas altoandinas evidencian el hecho de que Asteraceae y Poaceae se encuentran entre las familias dominantes (Smith, 1988; Flores et al., 2005; Cano et al., 2005, 2006, 2010, 2011; Paulino et al., 2015; Kahn et al., 2016, Aquino et al., 2017, 2018). Esto es debido a que muchas de sus especies han desarrollado características adaptativas a estos ecosistemas. En el caso de las Asteraceae el desarrollo de hojas pubescentes para reflejar la radiación, como en Baccharis tomentosa u hojas con el haz brillante para reflejar la luz y el envés pubescente, como en Gynoxys caracensis. Raíces profundas y flexibles para soportar los movimientos de las partículas del suelo por los cambios de temperatura, como en especies de Paranephelius. Crecimiento en forma de almohadilla o dentro de otras plantas en forma de almohadilla, como en especies de Werneria, y el desarrollo de espinas para protegerse de la depredación, como en Chuquiraga spinosa, que también permite a estas plantas adaptarse y ser dominantes en estos ecosistemas.

En relación a los géneros, tanto en el estudio de Quinteros (2009) como en el presente, se ha encontrado que Senecio y Baccharis presentan un mayor número de especies. Estos géneros también son los dominantes en otras provincias del Departamento de Lima como en Oyón (Paulino et al., 2015; Kahn et al., 2016), Huarochirí (Aquino et al., 2017, 2018), Canta (Gonzáles et al., 2015) y Yauyos (Trinidad \& Cano, 2016), así como en estudios de la familia Asteraceae en Lima (Gonzáles, 2016; Beltrán, 2018; Vilcapoma \& Beltrán, 2018). La evaluación florística realizada ha permitido catalogar un número importante de familias, géneros y especies en relación a otros trabajos realizados en las regiones de Ancash y Lima, tales como en el Distrito de San Marcos, Callejón de Conchucos y la Cordillera Blanca (Ancash), en el Distrito de Oyón y Matucana (Lima), y en el mismo Cajatambo (Lima) (Tabla 3). Cajatambo representa un área de alta riqueza de especies, lo cual constituye una fuente potencial de recursos vegetales para diversos usos, que podrían ser mejor aprovechados. Asimismo, existen especies comunes entre estas zonas de Lima: 173 especies en común con Huarochirí; 120 con Matucana; 112 con Yauyos; 77 con Oyón; y 77 con Canta, que representan el 34.3\%, 46.7\%, 39.7\%, 41.8\% y $17.0 \%$ de estas floras, respectivamente. Sin embargo, también se tienen 92 especies para Cajatambo que no están presentes en las zonas antes mencionadas, lo cual corresponde a un $26.2 \%$ de las especies registradas (Tabla 2). Estas especies representan un aporte en el conocimiento de la flora para la Región Lima. Asimismo, estos resultados refuerzan lo manifestado en otros estudios florísticos para la región en el sentido de tener un patrón similar para las zonas altoandinas en cuanto a las familias y géneros dominantes, pero que, sin embargo, debe seguir siendo documentada (Cano et al., 2010; Aquino et al., 2018) y la necesidad de seguir con estudios florísticos en lugares donde hay poca información (Kahn et al., 2016; Gonzáles, 2016; Aquino et al., 2018). La presente evaluación florística también ha permitido ampliar el conocimiento de la distribución de algunas especies, especialmente para Cajatambo; entre ellas, Baccharis pentlandii, Baccharis tomentosa, Bromus modestus, Calamagrostis grandis, Festuca glycerantha, Gentianella paludicola, Geranium matucanense, Gynoxys caracensis, Halenia umbellate, Jaltomata weberbaueri, Lachemilla orbiculata, Mycroseris pygmaea, Plantago myosuros, Senecio chiquianensis, Senecio hastifolius y Senna malaspinae; esta última endémica para Lima, colectada en la Expedición Malaspina en 1790, reportada luego en 1925 (Irwin \& Barneby, 1982) y que es de nuevo reportada recién en 1993 en Huamantanga, Canta (Flores, 1997).

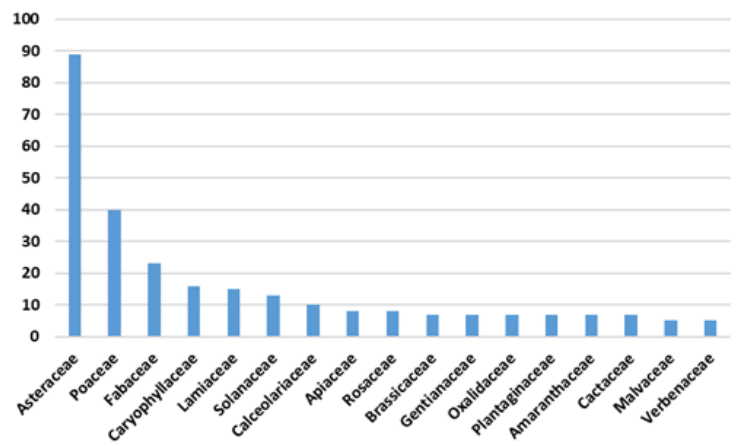

Figura 3. Familias con mayor número de especies en el Distrito de Cajatambo.

Estado de conservación de las plantas amenazadas

De las especies registradas se tiene que 31 de ellas presentan algún tipo de amenaza (Tabla 4 y Figura 4). Este conocimiento es muy importante debido a que la mayoría de estas especies son utilizadas por los cajatambinos como medicina, madera, leña, alimento, etc.; como Alnus acuminata "aliso", usada por su madera para tablas, vigas, muebles artesanales y leña; Austrocylindropuntia subulata "hualanca", como cerco vivo y su savia para la gastritis; Chuquiraga spinosa "quincha", como leña y desinflamante de la próstata; Polylepis microphylla "queñoa", por su madera para vigas, leña y elaborar cucharones y mazos; y Baccharis genistelloides "uclo", como forraje, por citar algunas de ellas (Benavides, 2019). El uso intensivo de estas especies podría estar creando una gran presión sobre ellas, por lo que, si no son manejadas de manera sostenible, por los pobladores, podría provocar su desaparición (Quinteros, 2009). También hay especies que están restringidas a localidades muy puntuales en Cajatambo, como Alnus acuminata en Ocopata, Juncus balticus en Iscu-Viconga, Senecio nivalis en Huaylashtoclanca, y Polylepis microphylla y Senecio rhizomatus en el cerro San Cristóbal. Asimismo, se ha 
registrado una mayor cantidad de especies amenazadas en algunas formaciones vegetales, tales como en matorral, en campo agrícola, en pajonal y en bosque de Polylepis, quizás debido a que en estos lugares se realizan diversas actividades agrícolas y pecuarias, pues las actividades antrópicas generan perturbaciones en el espacio y en el tiempo, alterando la estructura del ecosistema, de las comunidades o poblaciones y cambian la disponibilidad de recursos (Pickett \& White, 1985, citado por Castro, 2010). Estas formaciones vegetales podrían ser consideradas prioritarias en planes de conservación (Figura 5). Todo este conocimiento puede ayudar a tomar decisiones respecto a qué especies priorizar, qué localidades o zonas y en qué formaciones vegetales enfocar esfuerzos y recursos cuando se tengan que desarrollar planes de conservación y manejo sostenible del lugar. Finalmente, es importante considerar que las especies de cactáceas en la lista del presente trabajo están incluidas en el Apéndice II de la Convención sobre el Comercio Internacional de Especies Amenazadas de Fauna y Flora Silvestres (CITES, 2007). Estas especies, que no están necesariamente amenazadas de extinción, podrían llegar a estarlo, a menos que se controle estrictamente su comercio. Por ejemplo, Matucana haynei, Echinopsis peruviana y Austrocylindropuntia floccosa suelen ser extraídas de sus hábitats silvestres y vendidas como plantas ornamentales, medicinales o frutos, en muchos mercados de Lima (Vilcapoma, 2000; Ceroni \& Castro, 2013; MINAM, 2013). Estas plantas no son propagadas de manera artificial en condiciones controladas en viveros autorizados, tal como lo estipula el Artículo $279^{\circ}$ del Reglamento de la Ley No 27308 Ley Forestal y de Fauna Silvestre, aprobado por Decreto Supremo No 014-2001-AG (MINAGRI, 2001). Los usos de los cactus han generado un aumento en la demanda de algunas especies, especialmente en el extranjero, ya sea como plantas vivas, artesanías y algunos otros productos obtenidos a partir de ellos, por lo que se hace necesario acciones que promuevan su aprovechamiento sostenible y que no amenacen sus poblaciones naturales (MINAM, 2013).

\section{Conclusiones}

Se determinaron un total de 355 especies, correspondientes a 211 géneros y 64 familias. Las familias con mayor número de especies fueron: Asteraceae, Poaceae, Fabaceae, Caryophyllaceae, Lamiaceae y Solanaceae; mientras que los géneros con mayor número de especies fueron: Senecio, Baccharis, Calceolaria, Oxalis y Solanum.

Cajatambo representa un área de alta riqueza de especies y constituye una rica fuente de recursos vegetales para diversos usos.

Se encontraron 31 especies bajo alguna categoría de amenaza. Algunas especies están restringidas a localidades puntuales como Ocopata, Iscu-Viconga,
Huaylashtoclanca, y el cerro San Cristóbal. Las formaciones vegetales en donde más se ha registrado la presencia de especies amenazadas fueron: matorral, campo agrícola, pajonal y bosque de Polylepis.

El conocimiento de las especies vegetales de Cajatambo y los grados de amenaza, así como en qué localidades y formaciones vegetales específicas se encuentran, contribuirá en la toma de decisiones para el desarrollo de planes de conservación y manejo sostenible del lugar.

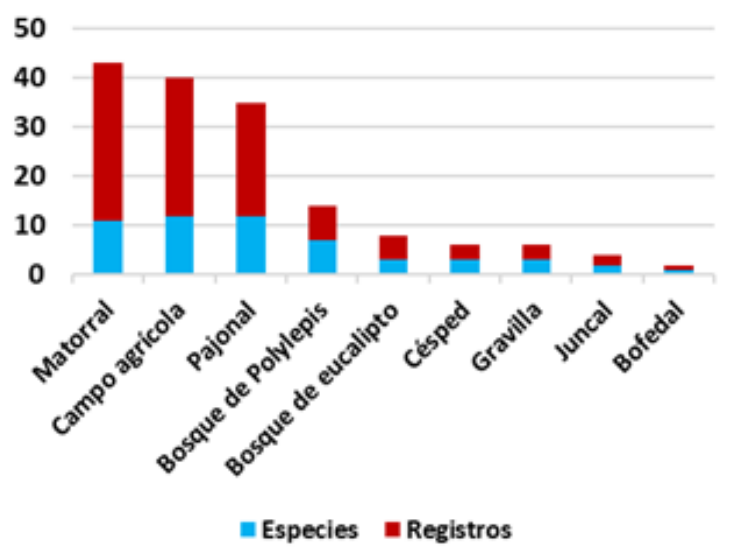

Figura 5. Número de especies amenazadas y registradas por tipo de formación vegetal, en el Distrito de Cajatambo, para el periodo 2015 - 2016.

\section{Agradecimientos}

Los autores expresan su agradecimiento al Proyecto FINCYT de INV. APLICADA, con código PIAP-2-P049-14; a la M.Sc. Mercedes Flores y al Sr. José Alegría por su apoyo en las determinaciones de especies de las familias Fabaceae y Poaceae; y al Herbario MOL (Herbario La Molina del Departamento Académico de Biología) de la Universidad Nacional Agraria La Molina.

\section{Literatura citada}

APG (Angiosperm Phylogeny Group). 2016. An update of the Angiosperm Phylogeny Group classification for the orders and families of flowering plants: APG IV. Botanical Journal of the Linnean Society, 181(1): 1-20. DOI: $10.1111 /$ boj.12385.

Aquino W., La Torre M., Condo F., Romero J. \& Ramírez J. 2017. Flora vascular del anexo de Marachanca del distrito de Matucana, provincia de Huarochirí, Lima, Perú. The Biologist, 15(2): 359-377. DOI: 10.24039/rtb2017152195.

Aquino W., Condo F., Romero J. \& Yllaconza R. 2018. Composición florística del distrito de Huarochirí, provincia de Huarochirí (Lima, Perú). Arnaldoa, 25 (3): 877-922. DOI: 10.22497/arnaldoa.253.25305.

Arnao L. 2017. Modelamiento de la estructura del paisaje relacionado con el cambio de temperatura para el distrito de Cajatambo entre 1987 - 2014. Tesis para optar el grado de Magister Science en Ecología Aplicada. Universidad Nacional Agraria La Molina. URI: http://repositorio.lamolina.edu.pe/handle/UNALM/4196. 
Beltrán H. 2018. Distribución y riqueza de Asteráceas en las cuencas hidrográficas del departamento de Lima, Perú. Arnaldoa, $25 \quad$ (3): $\quad 799-828 . \quad$ DOI: 10.22497/arnaldoa.253.25302.

Benavides C. 2019. Usos tradicionales de la diversidad vegetal: bases de la etnobotánica - en el distrito de Cajatambo, provincia de Cajatambo, Lima. Tesis para optar el título profesional de Bióloga. Universidad Nacional Agraria La Molina. Lima, Perú.

Brack A. \& Mendiola C. 2004. Ecología del Perú. Programa de las Naciones Unidas para el Desarrollo. Editorial Bruño. Versión en línea en: https://www.peruecologico.com.pe/libro.htm.

Cano A., La Torre M., Monsalve Ch., Roque J., Mendoza W., Salinas I., Castillo S. \& Aponte H. 2005. Las Plantas Comunes de San Marcos (Huari, Ancash). Guía de campo. Universidad Nacional Mayor de San Marcos. Museo de Historia Natural. Serie de divulgación $\mathrm{N}^{\circ} 12$.

Cano A., Castillo S., Aponte H., Morales M., Mendoza W., León B., Roque J., Salinas I., Monsalve Ch. \& Beltrán H. 2006. Las Plantas Comunes del Callejón de Conchucos (Ancash, Perú). Guía de campo. Universidad Nacional Mayor de San Marcos. Museo de Historia Natural. Serie de divulgación $\mathrm{N}^{\circ} 13$.

Cano A., Mendoza W., Castillo S., Morales M., La Torre M., Aponte H., Delgado A., Valencia N. \& Vega N. 2010. Flora y vegetación de suelos crioturbados y hábitats asociados en la Cordillera Blanca, Ancash, Perú. Revista Peruana de Biología, 17(1): 95-103. DOI: 10.15381/rpb.v17i1.56.

Cano A., Delgado A., Mendoza W., Trinidad H., Gonzáles P., La Torre M., Chanco M., Aponte H., Roque J., Valencia N. \& Navarro E. 2011. Flora y vegetación de suelos crioturbados y hábitats asociados en los alrededores del abra Apacheta, Ayacucho - Huancavelica (Perú). Revista Peruana de Biología, 18(2): 169-178. DOI: 10.15381/rpb.v18i2.224.

Castro S. 2010. Diversidad del hexápodo del suelo y caracterización de las parcelas en restauración tabacones (San Ignacio. Cajamarca). Tesis para optar el grado de Magister. Universidad Nacional Agraria La Molina. URI: http://repositorio.lamolina.edu.pe/handle/UNALM/ 1662.

Castro V. 2019. Efecto de la sucesión post-agrícola temprana sobre la diversidad vegetal en cuatro agroecosistemas en el distrito de Cajatambo, Lima, Perú. Tesis para optar el Grado de Doctor. Doctoris Philosophiae (Ph.D.). Universidad Nacional Agraria La Molina. URI: http://repositorio.lamolina.edu.pe/handle/UNALM/ 4196.

Ceroni A., Castro V., Teixeira V. \& Redolfi I. 2007. Neoraimondia arequipensis subsp. roseiflora (Werdermann \& Backeberg) Ostolaza (Cactaceae): eje de las interacciones en ecosistemas áridos. Revista Ecología Aplicada, 6(1 y 2): 155-168. DOI: 10.21704/rea.v6i12.352.

Ceroni A. \& Castro V. 2013. Manual de Cactus, Identificación y Origen. Ministerio del Ambiente. http://www.minam.gob.pe/diversidadbiologica/wpcontent/uploads/sites/21/2014/02/manual+de+cactus.co mpressed.pdf.
CITES (Convenio sobre el Comercio Internacional de Especies Amenazadas de Fauna y Flora Silvestres). 2017. Apéndices I, II y III del CITES. 4 oct. 2017. https://cites.org/sites/default/files/esp/app/2017/SAppendices-2017-10-04.pdf.

De la Cruz H., Zevallos P. \& Vilcapoma G. 2005. Status de conservación de las especies vegetales silvestres de uso tradicional en la provincia de canta, Lima-Perú. Revista Ecología Aplicada, 4(1,2): 9-16. DOI: 10.21704/rea.v4i12.292 .

Flores M. 1997. La Familia Leguminosae en el valle del Chillón - Parte media y alta. Departamento de Lima. Tesis para optar el grado de Magister. Universidad Nacional Mayor de San Marcos.

Flores M., Alegría J. \& Granda A. 2005. Diversidad Florística asociada a las Lagunas Andinas Pomacocha y Habascocha, Junín, Perú. Revista Peruana de Biología, 12: 125-134. DOI: 10.15381/rpb.v12i1.2366.

Font Quer P. 1965. Diccionario de Botánica. Editorial Labor S.A. España.

Gonzáles P., Navarro E., La Torre M.I. \& Cano A. 2015. Flora y vegetación del distrito de Santa Rosa de Quives, provincia de Canta (Lima). Arnaldoa, 22 (1): 155-182. http://journal.upao.edu.pe/Arnaldoa/article/viewFile/188 $/ 176$.

Gonzáles P. 2016. Riqueza y distribución de Asteraceae en el departamento de Lima (Perú). Arnaldoa, 23 (1): 111-134. http://200.62.226.189/Arnaldoa/article/viewFile/236/206

Gutiérrez J. \& Squeo F. 2004. Importancia de los arbustos en los ecosistemas semiáridos de Chile. Ecosistemas, Revista Científica de Ecología y Medio Ambiente, 13(1): 36-45.

https://www.revistaecosistemas.net/index.php/ecosistem as/article/view/215

Irwin H. \& Barneby R. 1982. The American Cassiinae. A Synoptical Revision of Leguminosae Tribe Cassieae subtribe Cassiinae in the New World. In: Irwin H.S. \& Barneby R.C. The American Cassiinae: a synoptical revision of Leguminosae tribe Cassieae subtribe Casiinae in the New World. Memoirs of the New York Botanical Garden, Vol 35, Part 1: 311-312. New York Botanical Garden. Bronx, N.Y. Versión en línea: http://mertzdigital.nybg.org/digital/collection/p9016coll 16/id/3899.

Kahn F., Millán B., Cano A., La Torre M., Baldeón S., Beltrán H., Trinidad H., Castillo S. \& Machahua M. 2016. Contribución a la flora altoandina del distrito de Oyón, región Lima, Perú. Revista Peruana de Biología 23(1): 67-72. DOI: $10.15381 / \mathrm{rpb} . v 23 i 1.11836$.

León B., Pitman N. \& Roque J. 2006. Introducción a las plantas endémicas de Perú. El libro rojo de las plantas endémicas de Perú. Revista Peruana de Biología,13(2): 922. DOI: $10.15381 /$ rpb.v13i2.1782.

Lot A. \& Chiang F. 1986. Manual de herbario. Administración y manejo de colecciones, técnicas de recolección y preparación de ejemplares botánicos. Departamento de Botánica / Instituto de Biología / UNAM / México.

Matteucci S. \& Colma A. 1982. Metodología para el estudio de la vegetación. Serie de Biología, monografía nro 22. Secretaría General de la Organización de los Estados Americanos. Washington, D.C. 
MINAGRI. 2001. Decreto Supremo No 014-2001-AG. Modifica el Reglamento de la Ley Forestal y de Fauna Silvestre. Ministerio de Agricultura y Riego. El Peruano (09/04/2001), XVIII(7328): 190283. Lima, Perú. http://minagri.gob.pe/portal/download/pdf/marcolegal/n ormaslegales/resolucionesministeriales/2013/mayo/modi f-reg-ley-forestal.pdf.

MINAGRI. 2006. Decreto Supremo $N^{\circ}$ 043-2006-AG. Aprueban Categorización de Especies Amenazadas de Flora Silvestre. Ministerio de Agricultura y Riego. El Peruano (13/07/2006), XXIII(9526): 323527. Lima, Perú. https://sinia.minam.gob.pe/download/file/fid/37595.

MINAM. 2013. "El San Pedro" o "Achuma". El género Echinopsis, Taxonomía, distribución y comercio. Informe de la Autoridad Científica CITES Perú. Ministerio del Ambiente. Disponible en: http://www.minam.gob.pe/diversidadbiologica/wpcontent/uploads/sites/21/2014/02/El-San-Pedro-oAchuma-g\%C3\%A9nero-Echinopsis.-

Taxonom\%C3\%ADa-disribuci\%C3\%B3n-yComercio.pdf

Mori S., Mattos L., Lisboa G. \& Coradin L. 1985. Manual de manejo do herbário fanerogamico. Centro de Pesquisas do Cacau. Ilhéus-Bahia.

ONERN (Oficina Nacional de Evaluación de Recursos Naturales). 1975. Inventario y evaluación de los recursos naturales de la zona del proyecto Marcapomacocha. Perú. Vol. 1. https://hdl.handle.net/20.500.12543/966.

Ostolaza C. 2014. Todos los cactus del Perú. MINAM (Ministerio del Ambiente). Lima, Perú. http://www.minam.gob.pe/diversidadbiologica/wpcontent/uploads/sites/21/2014/02/document.pdf. https://cdn.www.gob.pe/uploads/document/file/11962/d ocument.pdf.

Ostolaza C., Ceroni A., Dreyfus G., Calderón N. \& Novoa S. 2003. Cacti of the Huaura river valley, Perú - a conservation survey. British Cacti \& Succulent Journal, 21: 87-97. https://www.jstor.org/stable/42793888.

Ostolaza C., Ceroni A., Calderón N. \& Novoa S. 2005. The Huaura valley, Lima, Peru - revisited. British Cactus \& Succulent Journal, 23(1): 25-33. https://www.jstor.org/stable/42793996.

Ostolaza C., Ceroni A., Calderón N., Alvarez E., Zapata., Cortez J. \& Salinas L. 2006. Cacti of the Pativilca river basin, Lima, Peru. CactusWorld, 24(3): 117-128. BCSS. http://society.bcss.org.uk/index.php/journalcontents/2006-journal-contents.html.

Ostolaza C., Ceroni A., Zapata J., Cortez J., Salinas L. \& García E. 2007. Cacti of the Cañete river basin, Lima, Peru: a research and conservation study. CactusWorld, 25(4): 215-226.

BCSS. http://society.bcss.org.uk/index.php/journalcontents/2007-journal-contents.html.

Ostolaza C., Pino G., Flores M., Ceroni A., Ramírez N., Cortez J., Vargas J. \& Salinas L. 2009. Cacti of the Chancay and Chillón river basins, Lima, Peru: a research and conservation study. CactusWorld, 27(1): 39-50. BCSS. $\quad h t t p: / / s o c i e t y . b c s s . o r g . u k / i n d e x . p h p / 79-$ content/419-2009-journal-contents.html.
Paulino E., La Torre M. \& Huamán L. 2015. Distribución Altitudinal de la Flora Fanerogámica del distrito de Oyón, Lima, Perú. The Biologist, 13 (1): 21-33. DOI: http://dx.doi.org/10.24039/rtb2015131139.

Quinteros Y. 2009. Etnobotánica y revaloración de los conocimientos tradicionales de la flora medicinal en Cajatambo, Lima. Tesis para optar el grado de Magister en Desarrollo Ambiental. Escuela de Graduados. Pontificia Universidad Católica del Perú. URI: http://hdl.handle.net/20.500.12404/1580.

Ramos D., Castro V. \& Sánchez E. 2015. Caracterización de la vegetación a lo largo de una gradiente altitudinal en la comunidad de Cochahuayco, cuenca media del río Lurín, Lima. Ecología Aplicada, 14(1): 11-25. DOI: 10.21704/rea.v14i1-2.78.

Smith C. 1971. Preparing Herbarium Specimens of Vascular Plants. Agriculture Information Bulletin $\mathrm{N}^{\circ} 348$. Agricultural Research Service, U.S. Department of Agriculture.

https://naldc.nal.usda.gov/download/CAT87210188/PD F.

Smith D.N. 1988. Flora and Vegetation of the Huascaran National Park, Ancash, Perú, with preliminary taxonomic studies for a manual of the flora. Iowa State University. Ames,

Iowa. https://lib.dr.iastate.edu/cgi/viewcontent.cgi?article $=989$ $0 \&$ context $=\mathrm{rtd}$.

Trinidad H. \& Cano A. 2016. Composición florística de los bosques de Polylepis Yauyinazo y Chaqsii-Chaqsii, Reserva Paisajística Nor Yauyos-Cochas, Lima. Revista Peruana de Biología, 23(3): 271-286. DOI: 10.15381/rpb.v23i3.12862.

UICN (Unión Internacional para la Conservación de la Naturaleza). 2018. Lista Roja de Especies Amenazadas. Versión 2018-2 (en línea). Consultado 20 de febrero del 2019. Disponible en: http://www.iucnredlist.org/.

Vilcapoma G. 2000. Frutos silvestres de la cuenca del río Chillón (Cactaceae). Quepo 14: 59-67. https://specs.pe/publicaciones/ediciones-revista-quepo21.

Vilcapoma G. \& Beltrán H. 2018. Las asteráceas de la cuenca del río Chillón (Canta, Lima, Perú). Revista Ecología $\begin{array}{llll}\text { Aplicada } & 17 & \text { (2): } 171-184 . \quad \text { DOI: }\end{array}$ 10.21704/rea.v17i2.1237.

Young K. \& León B. 1990. Catálogo de la Zona Alta del Parque Nacional Rio Abiseo, Perú. Publicaciones del Museo de Historia Natural (UNMSM): Serie B (Botánica), 34: 1-37. https://museohn.unmsm.edu.pe/docs/pub_botanica/Publi caciones\%20Bot\%C3\%A1nica\%20Serie $\% 20 \mathrm{~B} \% 20 \mathrm{~N} \%$ C2\%B0\%2034.pdf.

Weberbauer A. 1945. El mundo vegetal de los Andes Peruanos. Estación Experimental Agrícola de La Molina / Ministerio de Agricultura / Perú. https://bibdigital.rjb.csic.es/idurl/1/9502. 
Tabla 1. Formaciones vegetales y transectos.

\begin{tabular}{|c|c|c|c|c|}
\hline Formación vegetal & Transecto & Coordenadas (utm) & Localidad & $\begin{array}{l}\text { Altitud } \\
\text { (msnm) }\end{array}$ \\
\hline \multirow{13}{*}{ Campo agrícola } & A1 & $\begin{array}{lll}18 \mathrm{~L} & 0279898 & 8841825\end{array}$ & Parientana & 3396 \\
\hline & $\mathrm{A} 2$ & 18L $0281266 \quad 8841058$ & Ocopata & 3470 \\
\hline & A3 & 18L $0280214 \quad 8841460$ & Ciscan & 3458 \\
\hline & A4 & $\begin{array}{lll}18 \mathrm{~L} & 0281228 & 8840365\end{array}$ & Ocopata & 3614 \\
\hline & A5 & $\begin{array}{lll}18 \mathrm{~L} & 0277407 & 8844245\end{array}$ & Urpaycocha & 2881 \\
\hline & A6 & $\begin{array}{lll}18 \mathrm{~L} & 0276401 \quad 8843493\end{array}$ & Tupicocha & 2993 \\
\hline & A7 & $\begin{array}{lll}18 \mathrm{~L} & 0278522 & 8842992\end{array}$ & Cruzgirca & 3230 \\
\hline & A8 & $\begin{array}{lll}18 \mathrm{~L} & 0280583 \quad 8846362\end{array}$ & Rancas & 3649 \\
\hline & A10 & 18 L $0282873 \quad 8840075$ & Tabin & 3564 \\
\hline & A11 & 18 L $0283238 \quad 8841093$ & Ambaroco & 3662 \\
\hline & A12 & 18L $0281213 \quad 8841403$ & Jagapampa & 3351 \\
\hline & A13 & 18L $0282293 \quad 8841450$ & Colca & 3482 \\
\hline & A14 & 18L $0277358 \quad 8843694$ & Puris & 2875 \\
\hline \multirow[t]{3}{*}{ Bosque } & Beu1 & $\begin{array}{llll}18 \mathrm{~L} & 0277698 & 8841392\end{array}$ & Entrada a Ciscan & 3472 \\
\hline & Beu2 & $\begin{array}{llll}18 \mathrm{~L} & 0280720 & 8842533\end{array}$ & Tizapampa & 3324 \\
\hline & Bpoly1 & $\begin{array}{lll}18 \mathrm{~L} & 0281417 & 8844120\end{array}$ & Cerro San Cristóbal & 4282 \\
\hline \multirow{12}{*}{ Matorral } & M1 & $\begin{array}{lll}18 \mathrm{~L} & 0276641 \quad 8842576\end{array}$ & Utcas & 3360 \\
\hline & M2 & $\begin{array}{lll}18 \mathrm{~L} & 0275415 & 8843237\end{array}$ & Cilcay & 3262 \\
\hline & M3 & 18L 02817968843064 & Cruce Uramasa Oyón & 3943 \\
\hline & M4 & 18L 02805558843340 & Cerca de la cantera & 3535 \\
\hline & M5 & 18L 02818728842968 & Cerca de la mina & 3844 \\
\hline & M6 & 18L $0278484 \quad 8843658$ & Cotaparaco & 3179 \\
\hline & M7 & 18L 02788698843061 & Cruzgirca & 3364 \\
\hline & M8 & 18L $0282289 \quad 8844206$ & Camino a Rancas & 3948 \\
\hline & M9 & 18L $0277120 \quad 8843908$ & Puris & 2802 \\
\hline & M10 & 18L 02798418842855 & Cashatambo & 3332 \\
\hline & M11 & 18L 02811998845969 & Puente Rancas & 3711 \\
\hline & M12 & 18L $0276178 \quad 8842683$ & Utcas & 3478 \\
\hline \multirow[t]{8}{*}{ Pajonal } & $\mathrm{P} 1$ & $\begin{array}{lll}18 \mathrm{~L} & 0282280 & 8843418\end{array}$ & La Punta & 4179 \\
\hline & $\mathrm{P} 2$ & 18L 02878918834402 & Tocanca & 4533 \\
\hline & P3 & 18L 02883658836782 & Huaylashtoclanca & 4367 \\
\hline & $\mathrm{P} 4$ & 18L 02871158839066 & Ucupy & 4373 \\
\hline & P5 & 18L 02853018842640 & Camino a Viconga & 4027 \\
\hline & P6 & $\begin{array}{lll}18 L & 0286643 & 8842933\end{array}$ & Iscu & 4065 \\
\hline & $\mathrm{P} 7$ & 18L $0280514 \quad 8839381$ & Milpoj & 3889 \\
\hline & P8 & $\begin{array}{lll}18 \mathrm{~L} & 0281848 \quad 8843865\end{array}$ & San Cristobal & 4245 \\
\hline \multirow[t]{3}{*}{ Césped } & $\mathrm{C} 1$ & 18L $0280347 \quad 8842758$ & Tizapampa & 3316 \\
\hline & $\mathrm{C} 2$ & 18L 02865798842984 & Iscu & 4006 \\
\hline & $\mathrm{C} 3$ & 18L $0281238 \quad 8839920$ & Camino a Milpoj & 3799 \\
\hline \multirow{2}{*}{ Bofedal } & B1 & 18L $0288337 \quad 8836726$ & Huaylashtoclanca & 4349 \\
\hline & $\mathrm{B} 2$ & 18L $0287097 \quad 8836938$ & Ucupy & 4192 \\
\hline \multirow[t]{2}{*}{ Gravilla } & G1 & 18L $0288103 \quad 8834323$ & Tocanca & 4534 \\
\hline & $\mathrm{G} 2$ & 18L $0288388 \quad 8834338$ & Tocanca & 4550 \\
\hline Juncal & $\mathrm{J} 1$ & 18L 02908228843980 & Iscu - Viconga & 4106 \\
\hline
\end{tabular}




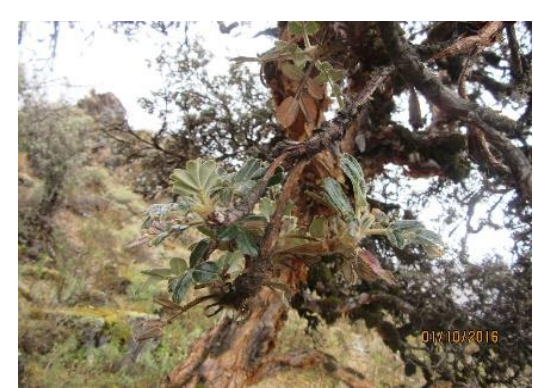

Polylepis microphylla "queñoa"

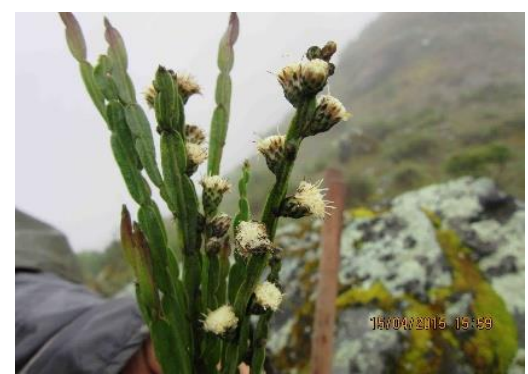

Baccharis genistelloides "uclo"

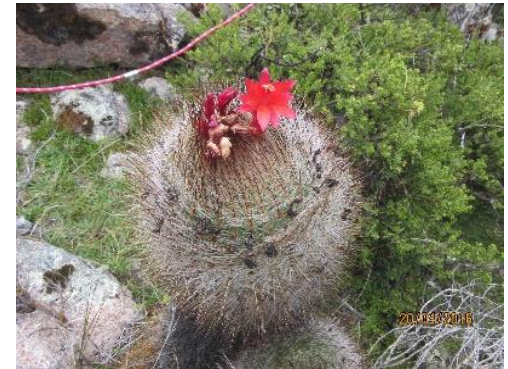

Matucana haynei

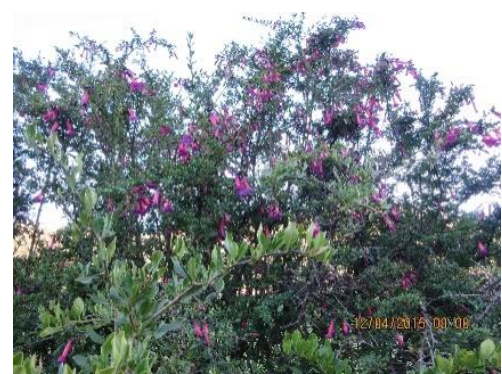

Cantua buxifolia "cantuta"

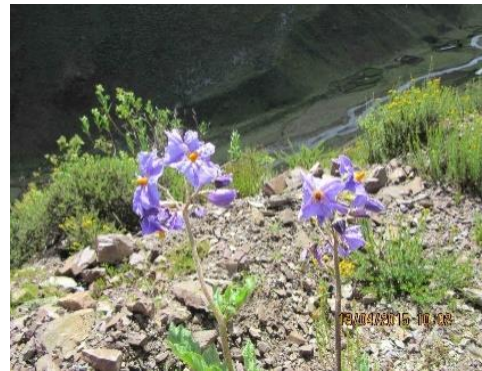

Solanun bukasovii

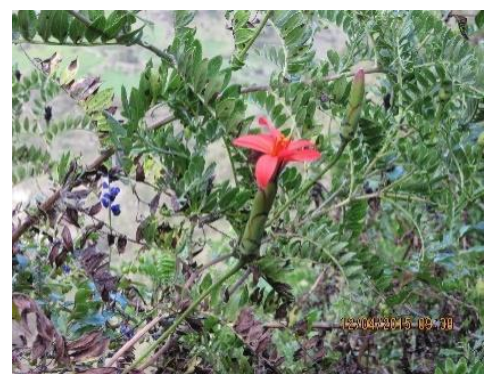

Mutisia acuminata "chinches"

Figura 4. Ilustraciones de algunas de las especies amenazadas en Cajatambo / Lima / Perú determinadas entre 2015 y 2016.

Tabla 2. Especies vegetales determinadas en el Distrito de Cajatambo para el periodo 2015 - 2016. FAMILIA / Género-especie

\section{ACANTHACEAE}

Dicliptera cf. acuminata (Ruiz \& Pav.) Juss. (*)

Dicliptera cf. montana Lindau (*)

Odontophyllum lyratum (Nees) Sreem.

\section{AGAVACEAE}

Agave cordillerensis Lodé \& Pino

Anthericum eccremorrhizum Ruiz \& Pav.

\section{ALSTROEMERIACEAE}

Bomarea cf. phyllostachya Mast. ex Baker (*)

Bomarea dulcis (Hook.) Beauverd

Bomarea involucrosa (Herb.) Baker

Bomarea ovata (Cav.) Mirb.

\section{AMARANTHACEAE}

Alternanthera cf. macbridei Standl. Alternanthera porrigens (Jacq.) Kuntze Amaranthus hybridus L.

Beta vulgaris $\mathrm{L}$.

Dysphania ambrosioides (L.) Mosyakin \& Clemants Gomphrena globosa L. (*)

Iresine lindenii Van Houtte (*)

\section{ANACARDIACEAE}

Schinus molle L.

\section{APIACEAE}

Arracacia incisa $\mathrm{H}$. Wolff

Azorella crenata (Ruiz \& Pav.) Persoon

Bowlesia cf. setigera H. Wolff (*)

Bowlesia lobata Ruiz \& Pav.

Conium maculatum L.

Cyclospermum leptophyllum (Pers.) Sprague ex Britton \& P. Wilson

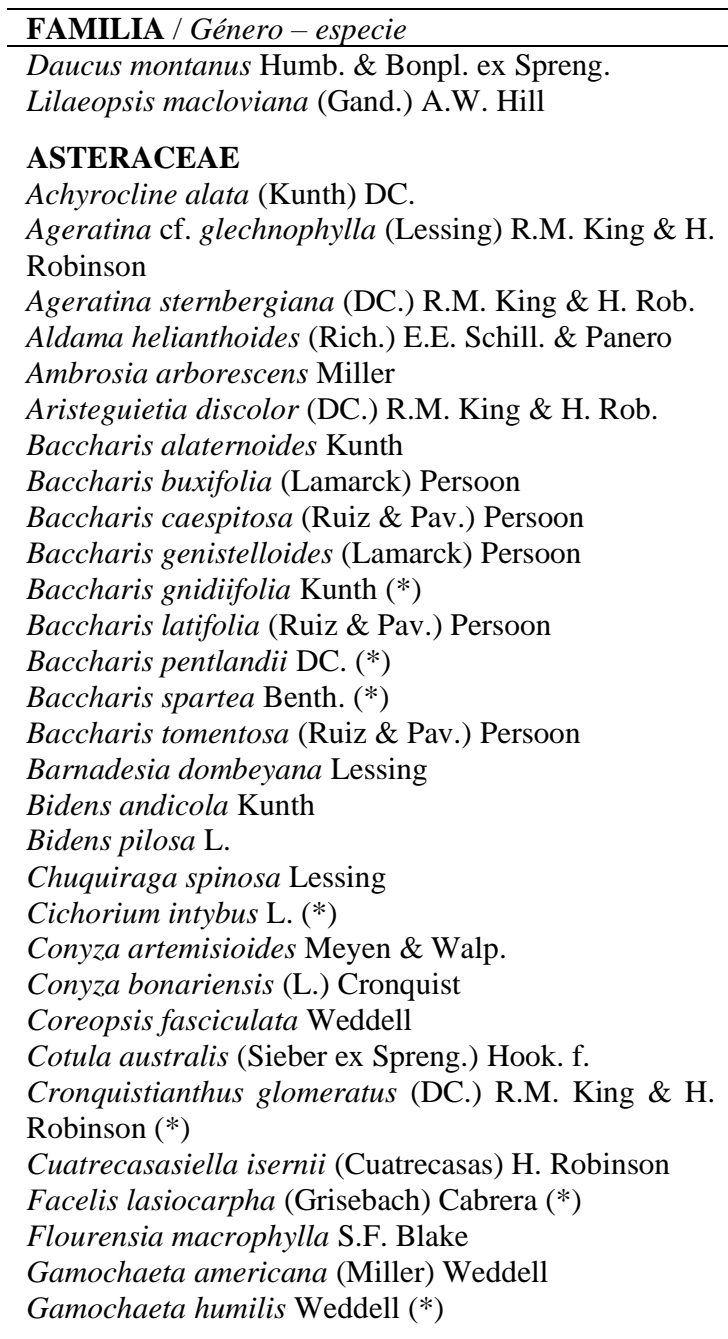




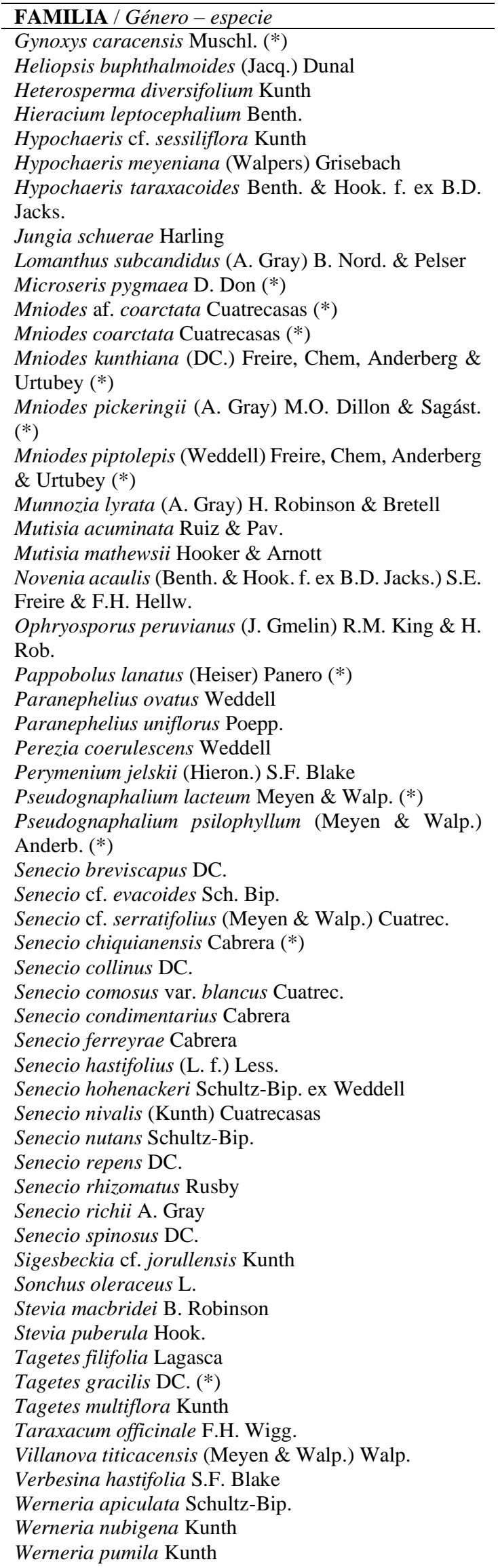

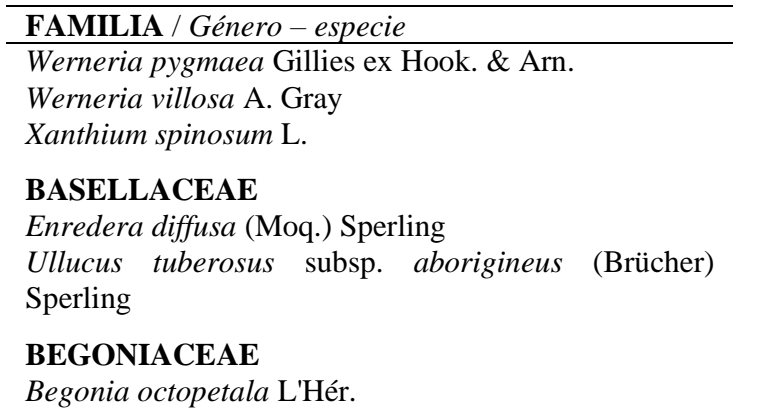

\section{BERBERIDACEAE}

Berberis cf. monosperma Ruiz \& Pav. (*)

Berberis lutea Ruiz \& Pav.

BETULACEAE

Alnus acuminata Kunth

\section{BIGNONIACEAE}

Tecoma stans subsp. sambucifolia (Kunth) J.R.I. Wood

\section{BORAGINACEAE}

Heliotropium macrostachyum (DC.) Hemsl. (*)

Plagiobothrys humilis (Ruiz \& Pav.) I.M. Johnst.

\section{BRASSICACEAE}

Brassica rapa ssp. campestris (L.) Peterm.

Capsella bursa-pastoris (L.) Medik.

Cardamine bonariensis Persoon

Lepidium bipinnatifidum Desv.

Lepidium chichicara Desv.

Nasturtium officinale W.T. Aiton

Sisymbrium cf. altissimum L. (*)

\section{CACTACEAE}

Armatocereus matucanensis Backeb. ex A.W. Hill Austrocylindropuntia floccosa (Salm-Dyck) F. Ritter Austrocylindropuntia subulata subsp. exaltata (Berger) Hunt

Borzicactus fieldianus subsp. tessellatus (Akers \& Buining) Krainz ex F. Ritter (*)

Echinopsis peruviana (Britton \& Rose) Friedrich \& G.D. Rowley

Loxanthocereus sulcifer Rauh \& Backeb. (*)

Matucana haynei (Otto ex Salm-Dyck) Britton \& Rose

\section{CALCIOLARIACEAE}

Calceolaria annua Edwin

Calceolaria aperta Edwin (*)

Calceolaria cuneiformis Ruiz \& Pav.

Calceolaria glauca Ruiz \& Pav.

Calceolaria lobata Cav.

Calceolaria scabra Ruiz \& Pav. (*)

Calceolaria scapiflora (Ruiz \& Pav.) Benth. (*)

Calceolaria tenuis Benth.

Calceolaria virgata Ruiz \& Pav.

Porodittia triandra (Cav.) G. Don

\section{CAMPANULACEAE}

Siphocampylus tupaeformis Zahlbr.

\section{CAPRIFOLIACEAE}

Valeriana globularis A. Gray

Valeriana interrupta Ruiz \& Pav.

Sambucus peruviana Kunth 


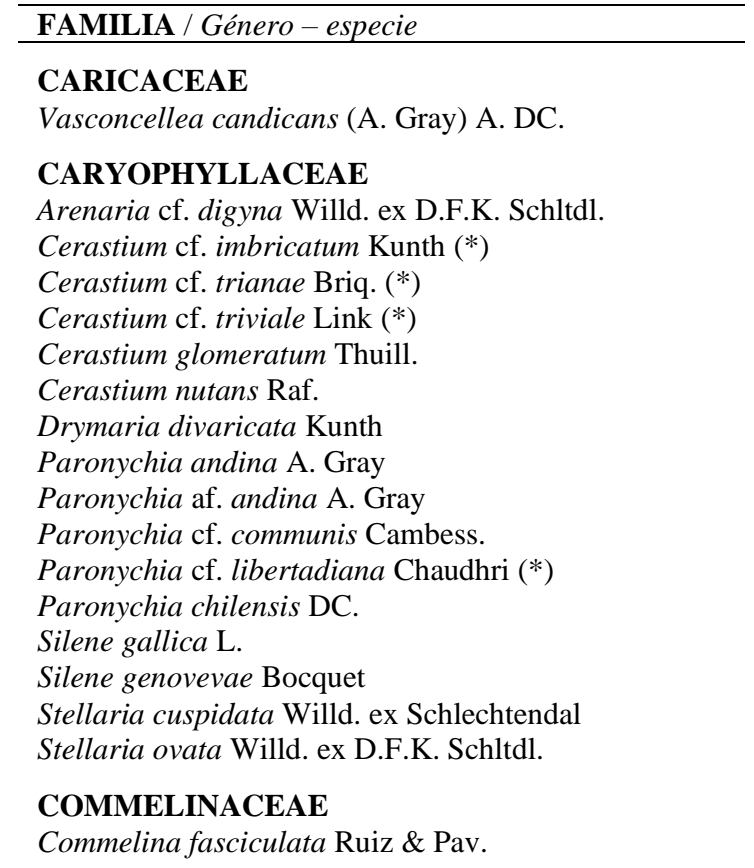

\section{CONVOLVULACEAE}

Dichondra microcalyx (Hallier f.) Fabris Ipomoea dumetorum Willd.

\section{CRASSULACEAE}

Echeveria cf. fruticosa Pino (*)

CUCURBITACEAE

Cyclanthera mathewsii Arn. ex A. Gray

CUSCUTACEAE

Cuscuta odorata Ruiz \& Pav. (*)

CYPERACEAE

Cyperus hermaphroditus (Jacq.) Standl. (*)

\section{EPHEDRACEAE}

Ephedra americana Humb. \& Bonpl. ex Willd. Ephedra rupestris Benth.

\section{EQUISETACEAE}

Equisetum bogotense Kunth

\section{ESCALLONIACEAE}

Escallonia resinosa (Ruiz \& Pav.) Persoon

\section{EUPHORBIACEAE}

Croton ruizianus Müll. Arg.

Euphorbia huanchahana (Klotzsch \& Garcke) Boiss. (*)

\section{FABACEAE}

Astragalus garbancillo Cav.

Dalea aff. exilis DC. $(*)$

Dalea cf. onobrychis DC.

Dalea exilis DC.

Dalea microphylla Kunth (*)

Dalea onobrychis DC.

Lathyrus magellanicus Lam.

Lupinus aff. ballianus C.P. Sm.

Lupinus ballianus C.P. Sm.

Lupinus microphyllus Desr.

Lupinus paniculatus Desr. (*)

Lupinus pygmaeus Tamayo ex Pittier (*)

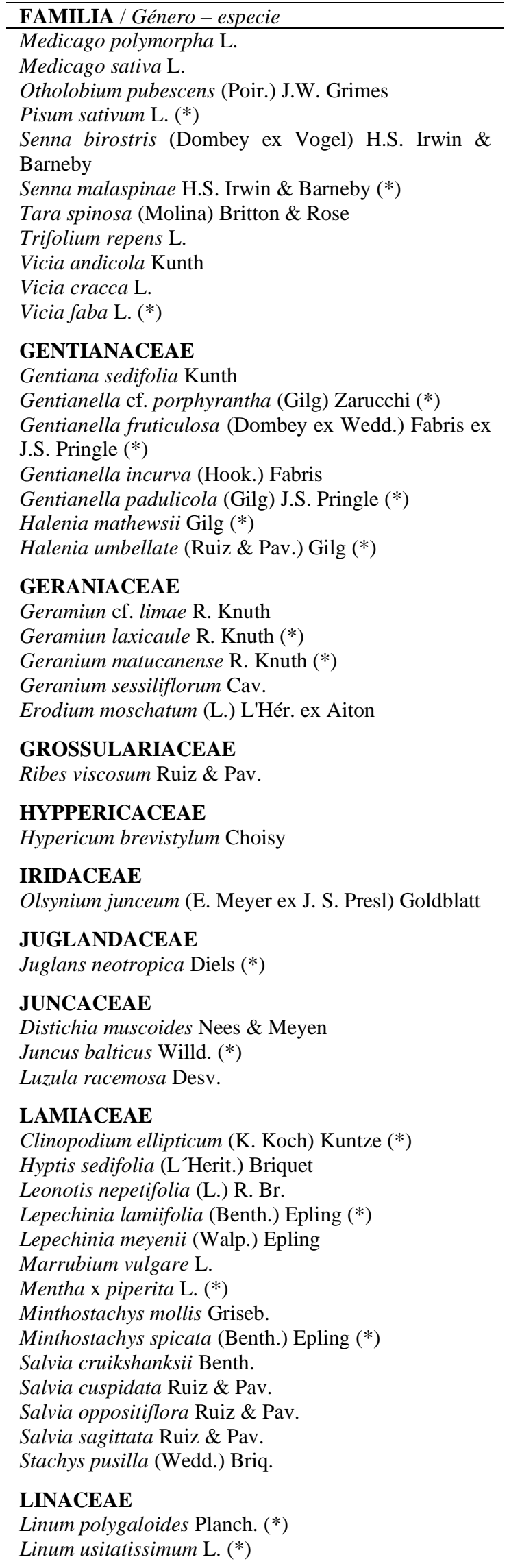




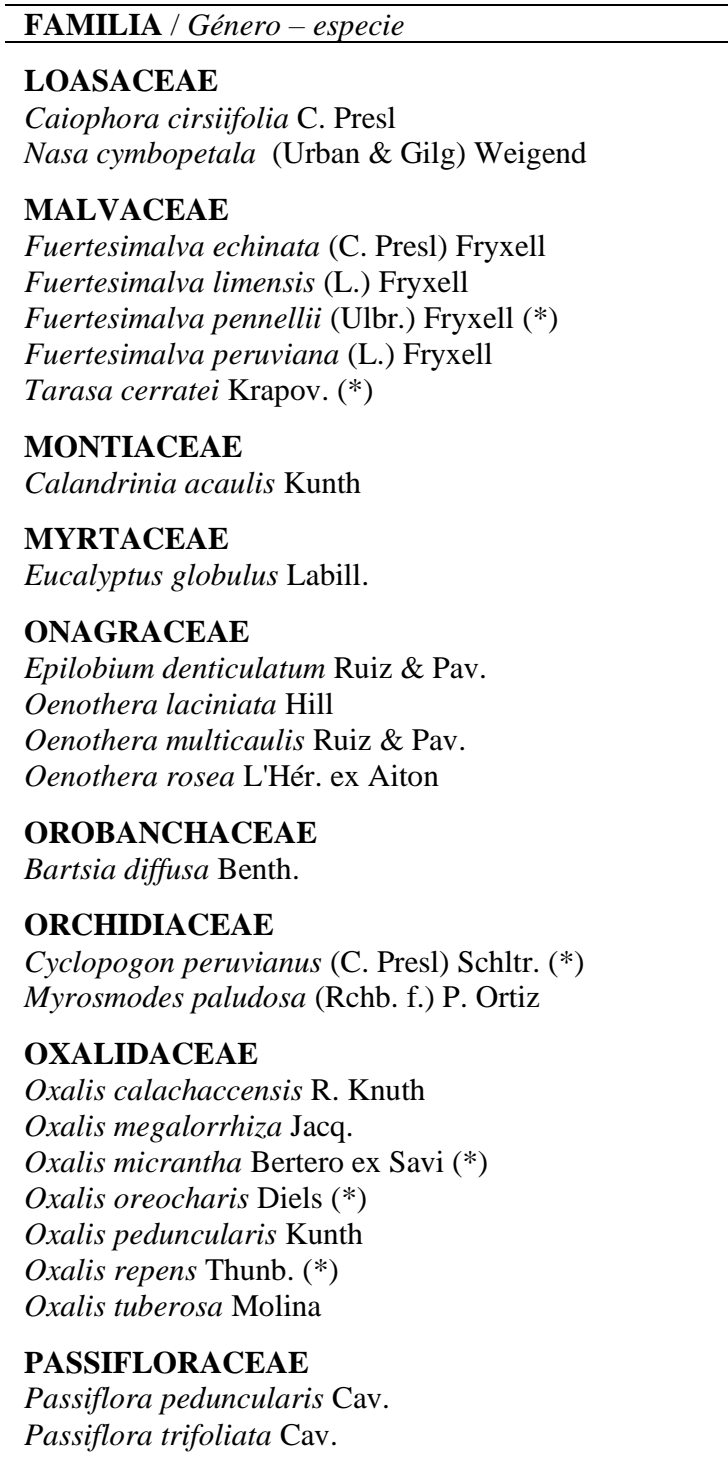

PHRYMACEAE

Mimulus glabratus Kunth

PIPERACEAE

Peperomia galioides Kunth

PLANTAGINACEAE

Plantago australis Lam.

Plantago lanceolata L.

Plantago linearis Kunth

Plantago myosuros Lam. (*)

Plantago rigida Kunth

Plantago sericea Ruiz \& Pav.

Veronica persica Poir.

\section{POACEAE}

Aciachne pulvinata Benth.

Agrostis breviculmis Hitchc.

Agrostis tolucensis Kunth

Avena sterilis L.

Bouteloua simplex Lag.

Brachypodium mexicanum (Roem. \& Schult.) Link (*)

Bromus catharticus Vahl

Bromus modestus Renvoize (*)

Calamagrostis eminens (J. Presl) Steud.

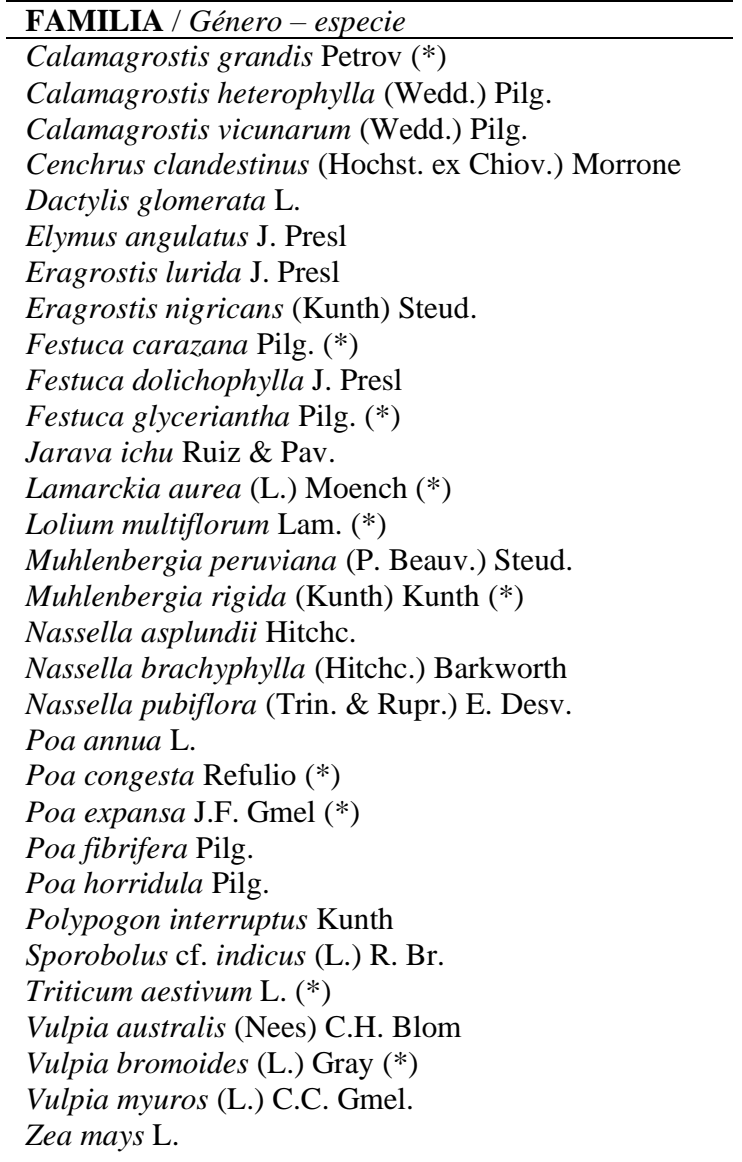

POLEMONIACEAE

Cantua buxifolia Juss. ex Lam.

POLYGALACEAE

Pteromonnina macrostachya (Ruiz \& Pav.) B. Eriksen Monnina salicifolia Ruiz \& Pav.

\section{POLYGONACEAE}

Muehlenbeckia volcanica (Benth.) Endl.

Polygonum aviculare L. (*)

Rumex obtusifolius L.

\section{PTERIDACEAE}

Adiantum capillus-veneris $\mathrm{L}$.

\section{RANUNCULACEAE}

Clematis cf. peruviana DC.

Ranunculus praemorsus Kunth ex DC.

Thalictrum longistylum DC.

\section{ROSACEAE}

Acaena torilicarpa Bitter

Hesperomeles cuneata Lindl.

Lachemilla cf. mandoniana (Wedd.) Rothm. (*)

Lachemilla diplophylla (Diels) Rothm.

Lachemilla orbiculata (Ruiz \& Pav.) Rydb. (*)

Lachemilla pinnata (Ruiz \& Pav.) Rothm.

Polylepis microphylla (Wedd.) Bitter (*)

Tetraglochin cristatum (Britton) Rothm.

\section{RUBIACEAE}

Arcytophyllum thymifolium (Ruiz \& Pav.) Standl. Galium aparine L. 


\section{FAMILIA / Género - especie}

\section{SCROPHULARIACEAE}

Alonsoa linearis (Jacq.) Ruiz \& Pav. Alonsoa meridionalis (L. f.) Kuntze

\section{SOLANACEAE}

Browallia cf. viscosa Kunth (*)

Cestrum auriculatum L'Hér.

Dunalia spinosa (Meyen) Dammer (*)

Jaltomata weberbaueri (Dammer) T. Mione \& F. G.

Coe $(*)$

Nicandra physalodes (L.) Gaertn.

Salpichroa ramosissima Miers (*)

Solanum acaule Bitter

Solanum aff. polytrichostylum Bitter (*)

Solanum basendopogon Bitter

Solanum bukasovii Juz. ex Rybin

\begin{tabular}{l}
\hline FAMILIA / Género - especie \\
\hline Solanum furcatum Dunal \\
Solanum tuberosum L. \\
URTICACEAE \\
Urtica flabellata Kunth \\
Urtica leptophylla Kunth \\
VERBENACEAE \\
Citharexylum cf. dentatum Tafalla ex D. Don \\
Citharexylum flexuosum (Ruiz \& Pav.) D. Don \\
Lantana scabiosiflora fo. albida Moldenke \\
Verbena cf. occulta Moldenke (*) \\
Verbena litoralis Kunth \\
\hline (*) Especies reportadas en el presente estudio que no \\
han sido reportadas en otros estudios florísticos en \\
Lima.
\end{tabular}

Tabla 3. Número de familias, géneros y especies en trabajos florísticos en Ancash y Lima.

\begin{tabular}{lccrl}
\hline Lugar & Familias & Géneros & Especies & \multicolumn{1}{c}{ Fuente } \\
\hline P.N. Huascarán (Ancash) & 104 & 340 & 779 & Smith (1988) \\
San Marcos (Huari, Ancash) & 25 & 49 & 57 & Cano et al. $(2005)$ \\
Callejón de Conchucos (Ancash) & 51 & 103 & 122 & Cano et al. (2006) \\
Cajatambo (Lima) & 56 & 133 & 200 & Quinteros (2009) \\
Oyón (Lima) & 40 & 126 & 184 & Paulino et al. (2015) \\
Santa Rosa de Quives (Lima) & 77 & 291 & 453 & Gonzáles et al. (2015) \\
Oyón (Lima) & 47 & 112 & 181 & Kahn et al. (2016) \\
R.P. Nor Yauyos-Cochas (Lima) & 60 & 172 & 282 & Trinidad \& Cano (2016) \\
Marachanca (Huarochirí, Lima) & 61 & 166 & 257 & Aquino et al. $(2017)$ \\
Huarochirí (Lima) & 84 & 296 & 504 & Aquino et al. (2018) \\
\hline
\end{tabular}


Tabla 4. Especies vegetales de Cajatambo con algún tipo de amenaza, según categorías propuestas por el Decreto Supremo 043-2006-AG (MINAGRI, 2016), la Unión Internacional para la Conservación de la Naturaleza (UICN, 2018), el Convenio sobre el Comercio Internacional de Especies Amenazadas de Fauna y Flora Silvestres (CITES, 2017) y Ostolaza (2014).

\begin{tabular}{|c|c|c|c|c|c|}
\hline Familia & Especie & ص & 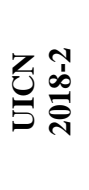 & $\begin{array}{c}\text { CITES } \\
2017\end{array}$ & 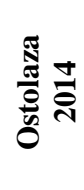 \\
\hline Asteraceae & Baccharis genistelloides & NT & & & \\
\hline Asteraceae & Chuquiraga spinosa & NT & & & \\
\hline Asteraceae & Mutisia acuminata & NT & & & \\
\hline Asteraceae & Perezia coerulescens & VU & & & \\
\hline Asteraceae & Senecio nivalis & VU & & & \\
\hline Asteraceae & Senecio nutans & VU & & & \\
\hline Asteraceae & Senecio rhizomatus & VU & & & \\
\hline Asteraceae & Werneria pumila & & $\mathrm{LC}$ & & \\
\hline Betulaceae & Alnus acuminata & VU & $\mathrm{LC}$ & & \\
\hline Bignoniaceae & Tecoma stan ssp. sambucifolia & NT & & & \\
\hline Brassicaceae & Nasturtium officinale & & $\mathrm{LC}$ & & \\
\hline Cactaceae & Armatocereus matucanensis & & $\mathrm{LC}$ & Apéndice II & NT \\
\hline Cactaceae & Austrocylindropuntia floccosa & & $\mathrm{LC}$ & Apéndice II & $\mathrm{LC}$ \\
\hline Cactaceae & Austrocylindropuntia subulata & & $\mathrm{LC}$ & Apéndice II & VU \\
\hline Cactaceae & Borzicactus fieldianus ssp. tessellatus & & $\mathrm{LC}$ & Apéndice II & EN \\
\hline Cactaceae & Echinopsis peruviana & & LC & Apéndice II & EN \\
\hline Cactaceae & Loxanthocereus sulcifer & & EN & Apéndice II & EN \\
\hline Cactaceae & Matucana haynei & VU & $\mathrm{LC}$ & Apéndice II & VU \\
\hline Caricaceae & Vasconcellea candicans & $\mathrm{CR}$ & & & \\
\hline Ephedraceae & Ephedra americana & NT & $\mathrm{LC}$ & & \\
\hline Ephedraceae & Ephedra rupestris & $\mathrm{CR}$ & $\mathrm{LC}$ & & \\
\hline Fabaceae & Medicago sativa & & $\mathrm{LC}$ & & \\
\hline Fabaceae & Tara spinosa & VU & & & \\
\hline Juglandaceae & Juglans neotropica & NT & EN & & \\
\hline Juncaceae & Juncus balticus & & $\mathrm{LC}$ & & \\
\hline Lamiaceae & Salvia oppositiflora & NT & & & \\
\hline Plantaginaceae & Plantago lanceolata & & VU & & \\
\hline Poaceae & Роа аппиа & & $\mathrm{LC}$ & & \\
\hline Polemoniaeae & Cantua buxifolia & NT & & & \\
\hline Rosaceae & Polylepis microphylla & & VU & & \\
\hline Solanaceae & Solanum bukasovii & VU & & & \\
\hline
\end{tabular}

\footnotetext{
${ }^{1}$ Jardín Botánico “Octavio Velarde Núñez”, Facultad de Ciencias, Universidad Nacional Agraria La Molina. Av. La Universidad s/n, La Molina, Lima, Perú. aceroni@lamolina.edu.pe.

${ }^{2}$ Herbario del Departamento Académico de Biología, Facultad de Ciencias, Universidad Nacional Agraria La Molina. Av. La Universidad s/n, La Molina, Lima, Perú. g_vilcapoma@ hotmail.com.
} 